\title{
Acupuncture Treatment for Symptom Management in Atopic Dermatitis: A Study Protocol for a Randomized, Participant- and Assessor-Blind, Sham-Controlled Trial
}

\author{
Jung Gun Park, ${ }^{1}$ Hi-Joon Park $\left(\mathbb{D},{ }^{2,3}\right.$ Younbyoung Chae $\mathbb{D}^{2,3}$ Yu-Kang Kim ${ }^{20},{ }^{2,3}$ \\ Hyangsook Lee $\mathbb{D}^{2,3}$ and Kyuseok Kim $\mathbb{D}^{4}$
}

${ }^{1}$ Department of Ophthalmology, Otorhinolaryngology and Dermatology of Korean Medicine, Graduate School of Korean Medicine, Kyung Hee University, Seoul 02447, Republic of Korea

${ }^{2}$ Department of Korean Medical Science, Graduate School, Kyung Hee University, Seoul 02447, Republic of Korea

${ }^{3}$ Acupuncture \& Meridian Science Research Centre, College of Korean Medicine, Kyung Hee University, Seoul 02447, Republic of Korea

${ }^{4}$ Department of Ophthalmology, Otorhinolaryngology and Dermatology of Korean Medicine, College of Korean Medicine, Kyung Hee University, Seoul 02447, Republic of Korea

Correspondence should be addressed to Hyangsook Lee; erc633@khu.ac.kr and Kyuseok Kim; kmdkskim@khu.ac.kr

Received 6 February 2019; Accepted 9 April 2019; Published 2 May 2019

Academic Editor: Keturah R. Faurot

Copyright (C) 2019 Jung Gun Park et al. This is an open access article distributed under the Creative Commons Attribution License, which permits unrestricted use, distribution, and reproduction in any medium, provided the original work is properly cited.

Atopic dermatitis (AD) is a chronic inflammatory skin disease with persistent itching, which impairs quality of life (QoL). Although various conventional treatments for $\mathrm{AD}$ exist, patients with $\mathrm{AD}$ often seek complementary and alternative therapies when conventional therapy has failed to relieve their AD symptoms or has had adverse effects. Acupuncture treatment may relieve AD symptoms, but controlled trials are needed to confirm this. Following our pilot study, which found that acupuncture treatment improves $\mathrm{AD}$ symptoms in mild-to-moderate $\mathrm{AD}$ patients, we will assess the effect of acupuncture treatment for symptom relief of $\mathrm{AD}$ using a trial with a complemented protocol. This is a two-arm, randomized, participant- and assessor-blinded, sham-controlled trial. A total of 36 mild-to-moderate $\mathrm{AD}$ patients will be randomly assigned in a 1:1 ratio to receive eight sessions twice weekly of either verum acupuncture (VA) or nonpenetrating sham acupuncture (SA) over four weeks. The primary outcome measured will be the change in the total Scoring Atopic Dermatitis (SCORAD) score. Secondary outcomes will be (1) changes in AD symptoms, QoL, dyspepsia symptoms, and electroencephalography (EEG) between baseline and week 4 and (2) changes in AD symptoms and QoL at baseline and at weeks 2, 4, and 8. This study will assess acupuncture treatment for the alleviation of AD symptoms in patients with mild-to-moderate AD. This clinical trial gas been registered in Korean Clinical Trial Registry (registration number: KCT0002796; date of registration: April 13, 2018).

\section{Introduction}

Atopic dermatitis (AD) is a chronic inflammatory skin disease characterized by intense itching, skin dryness, erythema, exudate, crust, and lichenification [1]. Globally, AD is a common health problem, and its incidence rate has increased 2-3 times in recent years in industrialized countries. Approximately, $20 \%$ of children and $3 \%$ of adults are suffering from $\mathrm{AD}[2,3]$.

Initial treatments for $\mathrm{AD}$ are emollients, topical corticosteroids, and topical calcineurin inhibitors $[4,5]$. Depending on severity, antihistamines, systemic corticosteroid, immunosuppressant, antibiotics, allergen-specific immunotherapy, or phototherapy may be used [6-8]. Complementary and alternative medicine treatments are used in patients with $\mathrm{AD}$, especially when there is no response to conventional therapies or side effects occurring [9-11].

$\mathrm{AD}$ has been effectively treated with acupuncture, which has been shown to lack side effects when used with conventional therapies [12-16]. In animal models, acupuncture treatment resulted in decreased skin overgrowth and pruritus and improved biomarkers $[5,17]$. To our knowledge, randomized 


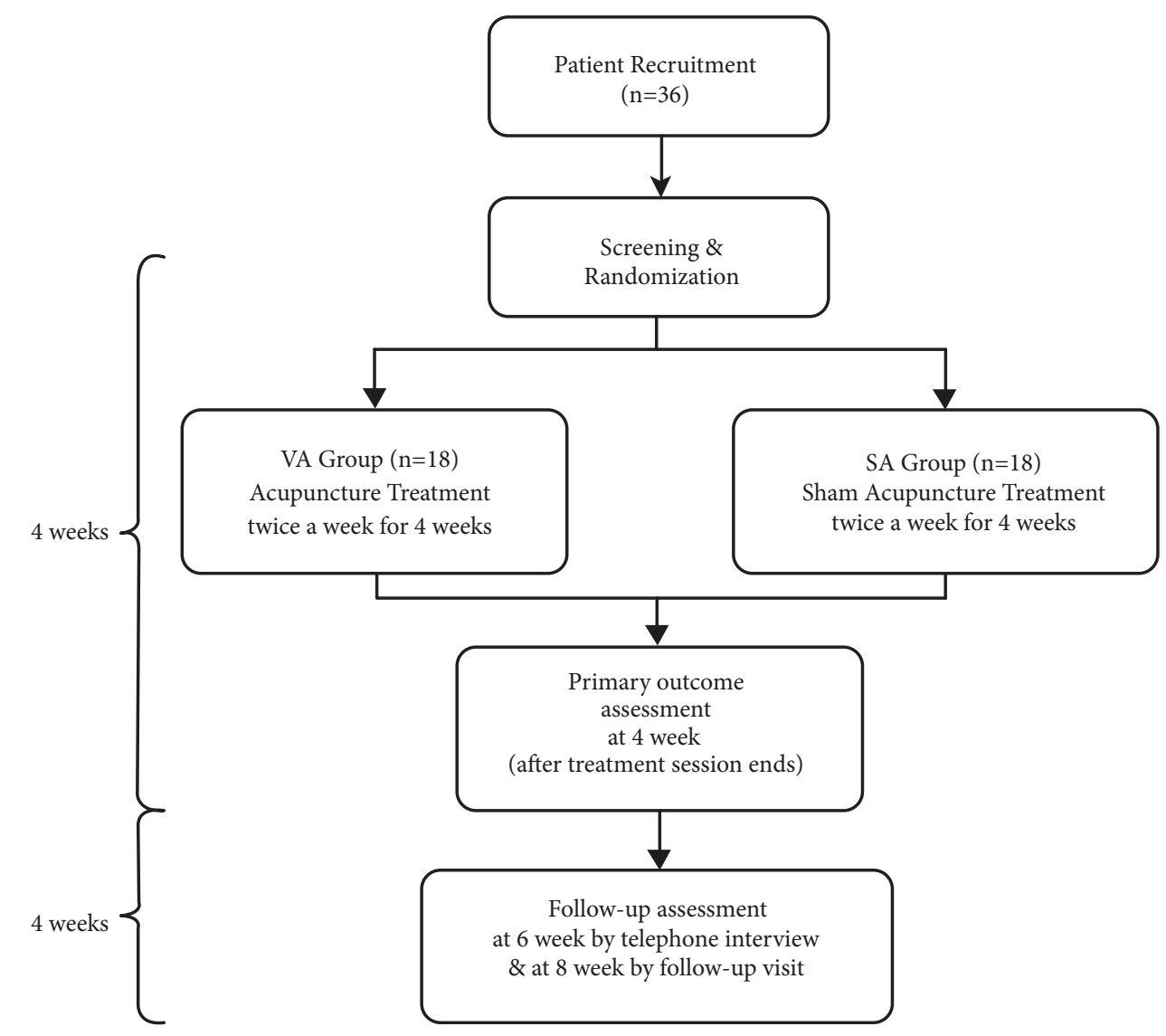

FIGURE 1: Flow of participants through the proposed trial. VA: verum acupuncture; SA: sham acupuncture.

controlled studies of the effectiveness of acupuncture-only treatment for AD are lacking [18], with the exception of our pilot study [19].

In this pilot study [19], a verum acupuncture (VA) treatment group showed significant alleviation of $\mathrm{AD}$ symptoms relative to the sham acupuncture (SA) group, including pruritus. Also, we found a positive relationship between the severity of AD symptoms and gastrointestinal (GI) symptoms such as epigastric tenderness and dyspepsia. Several studies show that GI and $\mathrm{AD}$ symptoms are related to unbalance of autonomic nerve system with sympathetic hyperactivity and parasympathetic dysfunction [20, 21]. Acupuncture treatment is known to affect autonomic nerve system [22]. Also, the relationship between $\mathrm{AD}$ and the central nervous system (CNS) has been well studied. Stress aggravates and sustains the itch-scratch cycle of AD by affecting the CNS [23]. Acupuncture treatment of $\mathrm{AD}$ patients may reduce brain activity of the insula, putamen, premotor and prefrontal cortical areas, which is induced by itching [24]. These findings can support the hypothesis of a "gut-brain-skin axis" suggesting a relationship between the GI tract, brain, and skin [25].

Taken together, this recent evidence suggests the need for a randomized controlled trial assessing the efficacy of acupuncture in reducing the symptoms of $\mathrm{AD}$. This study protocol describes the first such study. We designed a twoarm, randomized, participant- and assessor-blind, shamcontrolled trial based on our pilot study and the above recent studies. The trial aims to assess the effect of twiceweekly acupuncture treatment on the symptoms of mild-tomoderate $\mathrm{AD}$ in adults, including changes in quality of life (QoL), GI symptoms, and EEG.

\section{Methods}

2.1. Objectives. The main study objective is to determine whether or not twice-weekly acupuncture treatment improves symptoms and quality of life (QoL) in adults with mild-to-moderate AD.

Other objectives are (1) to investigate the relationship between severity of AD symptoms and GI symptoms and (2) to determine the effect of acupuncture on the CNS of $\mathrm{AD}$ patients using EEG.

2.2. Trial Design and Setting. This is a randomized, participant- and outcome assessor-blind, sham-controlled trial with a parallel group design. It will be conducted at the Kyung Hee University Korean Medicine Hospital. The flow chart of the study is presented in Figure 1. 


\subsection{Eligibility Criteria. Inclusion/exclusion criteria are as} follows.

2.3.1. Inclusion Criteria. (A) 19 to 65-year-old men and women able to read and write the Korean language

(B) AD diagnosed according to the Hanifin and Rajka criteria [26]

(C) Scores from 30 to 80 points on a $100 \mathrm{~mm}$ Visual Analog Scale (VAS) for pruritus (0, no symptom at all; 10, worst symptom ever) [27]

(D) Scores from 10 to 40 points on the objective Scoring Atopic Dermatitis (SCORAD) scale [28]

(E) Abstinence from prescribed medications for $\mathrm{AD}$ for the preceding month

(F) Agreement with a study protocol and willingness to sign written informed consent

(G) No participation in other clinical studies for the preceding month

\subsubsection{Exclusion Criteria. (A) Severely fluctuating AD symp-} toms

(B) Treatments (e.g., oral steroids) that may affect the symptoms of $\mathrm{AD}$ or that the study KMD deems inappropriate

(C) Secondary infection of AD lesions

(D) Severe mental problems

(E) Pregnancy, breastfeeding, or plans to have a baby during the study period

(F) Medical history that the study KMD considers to be inadequate

2.4. Recruitment of Participants. Based on our pilot study [19], we aim to recruit 36 mild-to-moderate AD patients using posters on noticeboards at the Kyung Hee Medical Center, nearby schools, and local community centers.

\subsection{Randomization and Allocation Concealment. Partici-} pants who meet the inclusion criteria and have signed the written informed consent will be randomly assigned to either the VA or the SA group in a 1:1 ratio. An independent statistician will generate a random number table using SAS 9.2 (PROC PLAN, SAS Institute Inc., Cary, NC, USA) and send it to the administrative staff at the Acupuncture and Meridian Science Research Center (AMSRC) at Kyung Hee University. The administrative staff, who are also independent of our research team, will put each random number in a sealed, sequentially numbered, opaque envelope. Once a participant passes screening, the investigator will call the AMSRC staff, who will immediately send the investigator a document containing that participant's unique random number and group allocation, which will be kept in the trial master file.

2.6. Blinding and Code Breaking. Participants and outcome assessors will be kept ignorant of treatment group allocations. It is not possible to blind the acupuncturist, so the KMD who applies the acupuncture treatment will not participate in assessment of trial outcomes or in collecting and analyzing the data. During acupuncture treatment, all participants in both groups will be shielded by a screen so that they cannot see the procedures. All participants in both groups will be treated with a Park sham device to ensure ignorance of whether the acupuncture needles penetrate the skin or not. If severe side effects occur or immediate acupuncture cessation is required, the blinding code will be broken.

\subsection{Interventions}

2.7.1. Acupuncture Treatment. Acupuncture treatment details are presented in Table 1 and are based on the revised Standards for Reporting Interventions in Clinical Trials of Acupuncture (STRICTA) 2010 checklist. Manual acupuncture (MA) and intradermal acupuncture (IA) will be performed for all participants in the VA group. All acupuncture treatments will be administered by one KMD with more than two years of clinical experience in Korean Medicine dermatology. This KMD will have undergone more than 10 hours of training and simulation to ensure that he is able to provide identical acupuncture treatment in accordance with the predefined protocol.

2.7.2. Verum Acupuncture (VA) Group. Participants in the VA group will receive eight acupuncture treatments twice a week for a total of four weeks. All VA group patients will receive acupuncture needling at six fixed acupoints. Up to 10 additional acupoints will be used, depending on each participant's daily general conditions. The six fixed acupoints are LI11, ST36, and PC6 bilaterally. Additional acupoints include ST43, GB41, LI2, TE3, SI3, TE6, SI2, BL66, LR3, SP3, ST43, and GB41 for gastric stuffiness or dyspepsia; LI2 and GB41 for tenderness around ST25, diarrhea, or constipation; TE3 and SI3 for fullness in the chest and hypochondrium; TE3 and TE6 for lower abdominal pain and tenderness on CV17; SI3 and GB41 for lower abdominal pain and dry skin; SI2 and BL66 for lower abdominal pain and heat in the upper body and cold in the lower body; and LR3 and SP3 for pain in the hypogastric region with darkness of the sublingual collateral vessels. Disposable sterile stainless needles $(0.25 \mathrm{x}$ $40 \mathrm{~mm}$; Dongbang Acupuncture Inc., Bundang, Seongnam, Korea) will be used. Acupuncture needles will be inserted to depths of 5 to $30 \mathrm{~mm}$, depending on the acupoint, and will remain in place for 15 minutes.

After removal of acupuncture needles, three acupoints will be treated with IA using $1.5 \mathrm{~mm}$ press tack needles (Haeng Lim Seo Won Medical Co., Korea). These are bilateral LI11 and auricular-Shenmen contralaterally to the side with more severe symptoms. Participants will be instructed by the study KMD to press LI11 for more than three minutes when they experience severe itching.

2.7.3. Sham Acupuncture (SA) Group. Participants in the SA group will receive sham acupuncture treatment eight times twice a week for four weeks. Unlike the VA group, SA group patients will receive sham acupuncture treatment at only six fixed control points. These bilateral acupoints are as follows: 1 to $2 \mathrm{~cm}$ proximal and $1 \mathrm{~cm}$ medial to LI7, $1 \mathrm{~cm}$ proximal and $1 \mathrm{~cm}$ medial to LI11, and $1 \mathrm{~cm}$ proximal and $1 \mathrm{~cm}$ lateral 


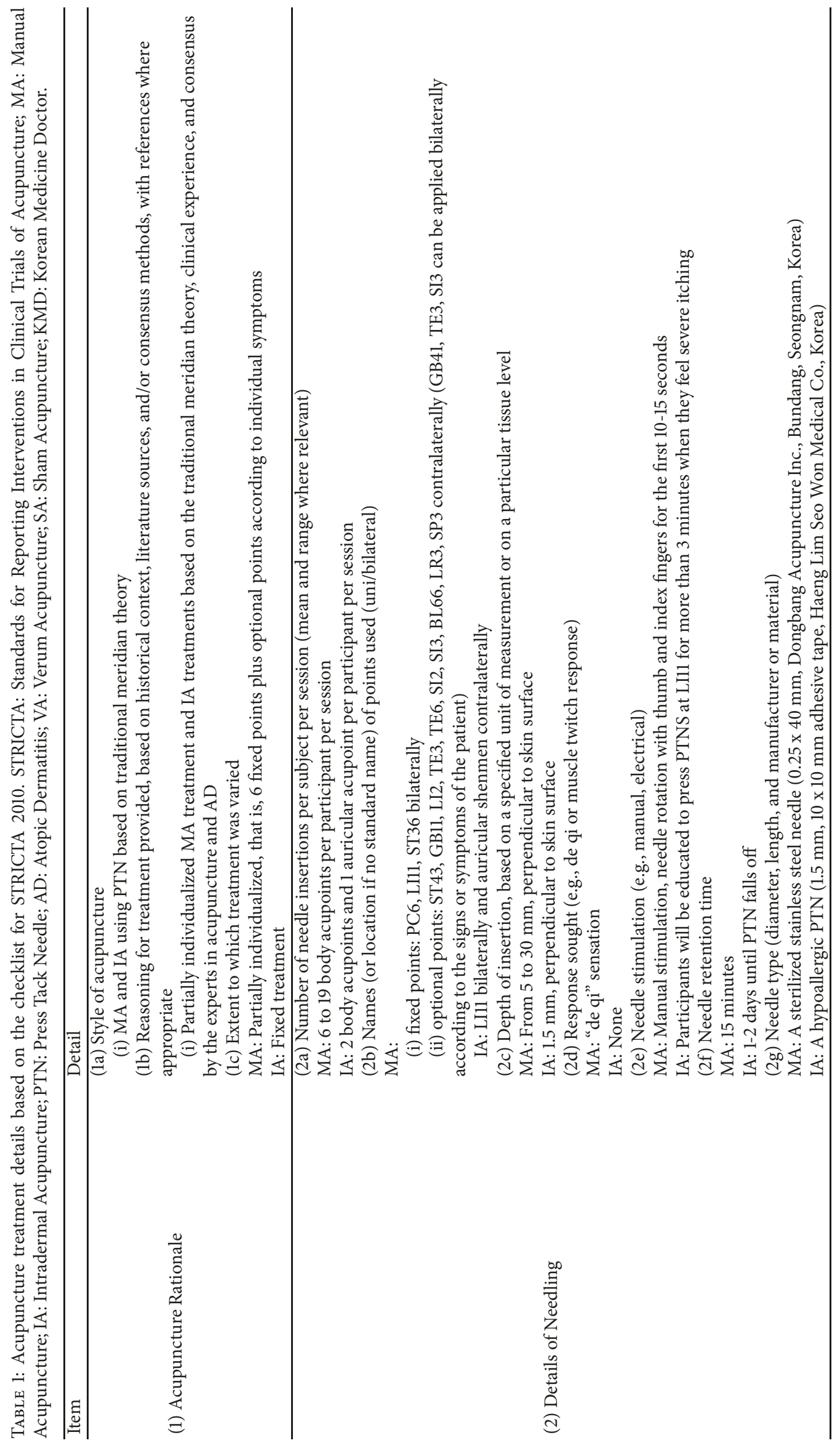




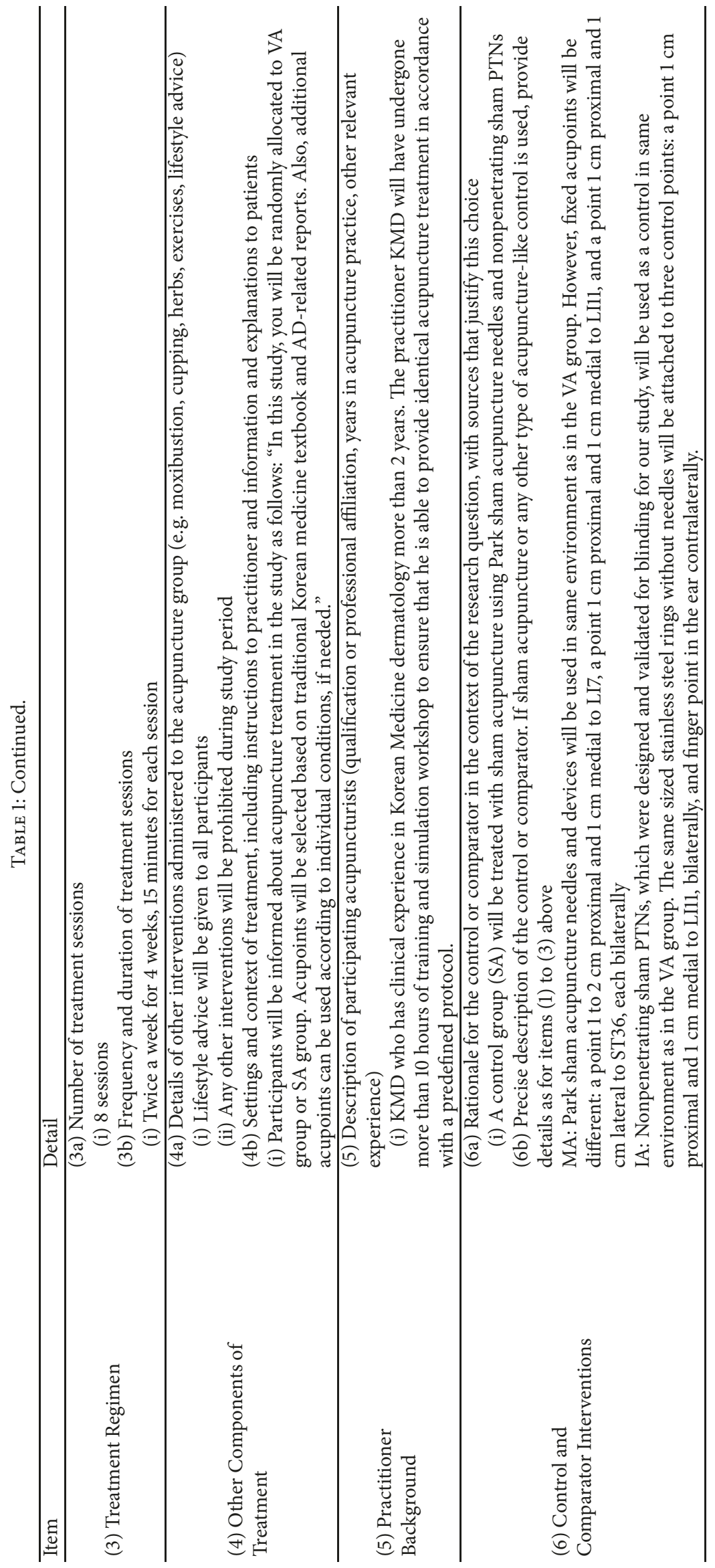


TABle 2: Study Schedule. VAS: Visual Analog Scale; SCORAD: Scoring Atopic Dermatitis; EASI: Eczema Area and Severity Index; POEM: Patient Oriented Eczema Measure; DLQI: Dermatology Life Quality Index; PPT: Pain Pressure Threshold; NDI-K: Nepean Dyspepsia IndexKorean version; AR: Adequate Relief of functional dyspepsia pain and discomfort; EEG: Electroencephalography. represents treatment applied at that particular interval; $\boldsymbol{O}$ : participant contacted by telephone.

\begin{tabular}{|c|c|c|c|c|c|c|c|c|c|}
\hline \multirow{2}{*}{ Treatment and measure } & \multirow{2}{*}{ Baseline } & \multicolumn{4}{|c|}{ Treatment period } & \multicolumn{4}{|c|}{ Follow-up period } \\
\hline & & 1 week & 2 weeks & 3 weeks & 4 weeks & 5 weeks & 6 weeks & 7 weeks & 8 weeks \\
\hline Demographics & - & & & & & & & & \\
\hline Physical Examination & - & & & & & & & & \\
\hline Medical history & - & & & & & & & & \\
\hline VAS for itch & $\bullet$ & - & ○ & ○ & ○ & & 8 & & - \\
\hline VAS for sleep loss & 0 & 0 & 0 & $\bullet$ & 0 & & 8 & & 0 \\
\hline VAS for dyspepsia & 0 & 0 & $\bullet$ & $\bullet$ & 0 & & 8 & & $\bullet$ \\
\hline SCORAD assessment & $\bullet$ & $\bullet$ & $\bullet$ & $\bullet$ & $\bullet$ & & & & $\bullet$ \\
\hline EASI assessment & & $\bullet$ & $\bullet$ & $\bullet$ & $\bullet$ & & & & $\bullet$ \\
\hline POEM assessment & & $\bullet$ & $\bullet$ & $\bullet$ & $\bullet$ & & & & $\bullet$ \\
\hline DLQI assessment & & $\bullet$ & $\bullet$ & $\bullet$ & $\bullet$ & & & & $\bullet$ \\
\hline PPT evaluation & & $\bullet$ & $\bullet$ & & $\bullet$ & & & & \\
\hline NDI-K assessment & & $\bullet$ & & & $\bullet$ & & & & $\bullet$ \\
\hline AR assessment & & & & - & 0 & & 0 & & 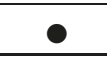 \\
\hline Credibility test & & $\bullet$ & & & 0 & & & & \\
\hline Blinding test & & & & & $\bullet$ & & & & \\
\hline EEG assessment & & $\bullet$ & & & $\bullet$ & & & & \\
\hline Acupuncture Treatment & & 0 & 0 & 0 & 0 & & & & \\
\hline Adverse Events & & $\bullet$ & $\bullet$ & $\bullet$ & - & & 0 & & - \\
\hline
\end{tabular}

to ST36. To maintain participant blinding, a nonpenetrating sham device (Park sham acupuncture needles, AcuPrime Co., Ltd., Exeter, UK) will be used and left in place for 15 minutes.

After removal of sham acupuncture needles, the IA procedure used in the VA group will be replaced with application of needle-free, stainless steel rings that do not penetrate the skin. These will be attached to three control points: $1 \mathrm{~cm}$ proximal and $1 \mathrm{~cm}$ medial to LI11 bilaterally and finger point in the ear contra-laterally to the side with more severe symptoms. Unlike the VA group, SA group patients will not be instructed to press the control point.

2.7.4. After Treatment. Two weeks after the eighth visit for the final acupuncture treatment, a telephone interview will be conducted to assess the severity of AD and dyspepsia symptoms, side effects after acupuncture treatment, and whether or not other treatments were used during the trial. During a final visit, four weeks after the eighth visit, the participant will complete a final evaluation. No other treatments will be allowed during the study period.

2.8. Compliance and Discontinuation. Frequent hospital visits may be a burden to the participant, resulting in withdrawal from the study. Based on the results of our pilot study [19], we determined that completion of six to eight treatment sessions was sufficient for a participant to be considered compliant and yield acceptable data. For dropouts who do not fulfil this criterion, the last observed data will be used for the analysis. The research coordinator will keep in contact with participants to encourage them to continue participation to the end of the trial.

2.9. Outcome Measures. A researcher who is independent of this study will evaluate the outcomes. The schedule is shown in Table 2. The primary measurable outcome is the change in SCORAD (total) before treatment (baseline) and after (at four weeks) Treatment. Secondary outcomes are as follows: (1) baseline to four-week changes in AD symptoms, QoL, GI symptoms, or EEG and (2) changes between baseline and second, fourth, and eighth weeks in AD symptoms and QoL. Measurable values are as follows: SCORAD Index, Eczema Area and Severity Index (EASI), Patient Oriented Eczema Measure (POEM), Dermatology Life Quality Index (DLQI), abdominal Pressure Pain Threshold (PPT) using an algometer developed in the Korea Institute of Oriental Medicine [29], questionnaires about dyspepsia symptoms (Nepean Dyspepsia Index-Korean version, Adequate Relief of functional dyspepsia pain and discomfort, and VAS for dyspepsia), and EEG.

2.9.1. Scoring Atopic Dermatitis (SCORAD). SCORAD was developed in 1993 by the European Task Force on Atopic Dermatitis as a useful scoring system that assesses the severity of $\mathrm{AD}$ by its extent, intensity, and subjective symptoms [28, $30,31]$. The extent component of SCORAD is assessed using the rule of nines for AD lesions. The intensity component 
of SCORAD is divided into erythema, edema and papules, oozing and crust, excoriation, lichenification, and dryness. Subjective symptom measures are the degrees of pruritus and sleep disturbance assessed on the VAS.

2.9.2. Eczema Area and Severity Index (EASI). EASI is a tool for evaluating the severity of $\mathrm{AD}$ symptoms. Like the SCORAD index, it is known as a useful evaluation tool [30, 32,33]. Body parts are divided into four parts (head and neck, trunk, upper limb, and lower limb) to evaluate the extent of $\mathrm{AD}$ lesions. The severity of symptoms (erythema, edema and papules, excoriation, and lichenification) is then evaluated.

\subsubsection{Patient Oriented Eczema Measure (POEM). POEM} is a simple, easily understood, and well-validated tool for evaluating the severity of $\mathrm{AD}$ symptoms in the preceding week. It is known to be appropriate for use in outpatient clinic or clinical trial settings $[30,34]$.

2.9.4. Dermatology Life Quality Index (DLQI). DLQI is a wellvalidated clinical research tool that is widely used to assess the QoL of patients with dermatologic symptoms. It evaluates the effect of skin disease on QoL during the preceding week and consists of 10 questions $[35,36]$.

\subsubsection{Abdominal Examination Using Pain Pressure Threshold} $(P P T)$. In Korean Medicine, KMDs use abdominal examination for diagnosis, choice of treatment methods, and/or evaluation of prognosis [37]. We will standardize and describe abdominal examination quantitatively using the PPT algometer. The PPT algometer used in our pilot study [19] was found to be unsuitable for abdominal examination, so a more appropriate algometer developed in the Korea Institute of Oriental Medicine will be used [29]. The outcome assessor will apply the algometer before and after acupuncture treatment to measure the patient's abdominal tenderness at four acupuncture points (CV17, CV12, and bilateral ST25). Pressure at each acupoint will be delivered at a constant force and velocity following the computerized guide. Each measurement will be performed three times for 1 minute [38].

2.9.6. Nepean Dyspepsia Index (NDI). NDI is a reliable scale for functional dyspepsia which evaluates dyspepsia symptoms and related QoL. NDI consists of period, severity, and degree of distress of 15 dyspepsia-related symptoms [39, 40]. In this study, participant will be asked to answer the Korean version of NDI (NDI-K) [41].

2.9.7. Adequate Relief of Functional Dyspepsia Pain and Discomfort (AR). AR assesses improvement in overall functional dyspepsia symptoms [42]. Subjects will be asked to answer the following question: "Have you experienced appropriate relief (more than 50\% reduction) of the pain or discomfort associated with your dyspepsia after the last acupuncture treatment?"
2.9.8. VAS for Functional Dyspepsia. Using $100 \mathrm{~mm}$ VAS (0 $\mathrm{mm}$ for no discomfort to $100 \mathrm{~mm}$ for the worst discomfort), we will measure a degree of overall dyspeptic symptoms.

2.9.9. Credibility/Blinding Test. In order to evaluate the credibility of the study participants' acupuncture treatment, a questionnaire-type credibility test will be conducted at the first and eighth treatment visits and the follow-up visit [43]. The following question will also be asked once at the eighth treatment visit when the last treatment is given: "Which group do you think you belonged to? The treatment group or the control group, and why?" This question aims to determine the direction and degree of blinding between the VA group and the SA group [44].

2.9.10. Measurement of Electroencephalography (EEG). Subjective feelings of itchiness in $\mathrm{AD}$ may be induced or enhanced by watching someone scratch himself or herself. Brain signals associated with somatosensory perception may be changed by contagious itch [45]. Visual stimuli depicting scratching behavior can induce changes in oscillatory potentials measured with EEG. This study is designed to explore the relationship between neuronal oscillations and subjective itchiness induced by contagious itch.

All patients will be asked to evaluate how much itchiness they feel after watching video clips of a person either scratching or performing a control movement. Their evaluation will use a visual analog scale ranging from 0 to 10 . Continuous EEG will be recorded using Brain Products EEG amplifiers with a 32-channel ActiCAP system according to the international 10-20 system. Event-related spectral perturbation (ERSP) methods will be applied to EEG data, while patients are watching the video clips. Through time-frequency analysis, the suppression of mu activity will be measured over central-parietal regions. $\mathrm{Mu}$ rhythm represents frequencies in the range of $8-13 \mathrm{~Hz}$, and the suppression of mu activity can be observed over the primary sensorimotor cortex when an action is performed, observed, or imagined [46]. The EEG results will be compared within each patient between scratching and control movement videos. We hypothesize that the suppression of mu activity over the sensorimotor cortex will be decreased after the treatment in the VA group but not in the SA group.

2.10. Sample Size Calculation. Sample size calculation was carried out using $G *$ Power 3.1.9.2 for Windows 10. The primary measurable outcome in this study is the change in SCORAD (total) before treatment (at baseline) and at the end of treatment (four weeks). We derived the sample size of this study using information from our pilot study [19] showing a change in total SCORAD at baseline and after treatment of $13.0 \pm 9.9$ and $4.6 \pm 5.0$ in the VA and SA groups, respectively, and effect size $d$ was 1.1. Based on this, the critical t-value was 2.0 for 28 degrees of freedom, and the noncentrality parameter $\delta$ was 3.0 (two-tailed independent $t$-test) at $5 \%$ significance level, with $80 \%$ power and $20 \%$ dropout rate. Required sample size was calculated as 18 per group. 
2.11. Statistical Analysis. Statistical analyses will be performed by an independent statistician who is unaware of group assignments, using IBM SPSS for Windows (Version 21.0). Continuous variables will be presented as mean \pm 1 standard deviation, and bivariate variables will be presented as numbers (\%). The effect of the intervention will be assessed as the change in outcome measures between baseline and four weeks (after treatment sessions end) and through eight weeks (after follow-up period ends), using a repeated-measures analysis of variance (ANOVA) and $\chi 2$ test. The difference in effect between VA and SA will be assessed by comparing changes in the SCORAD (total) index, the AD symptoms, QoL, dyspepsia symptoms, and EEG outcomes, using the independent $t$-test or Mann-Whitney $U$ test on continuous variables and $\chi 2$ test or Fisher's exact test on categorical variables.

ITT (intention-to-treat) analysis will be used for all participants who have undergone at least one acupuncture treatment. For missing values, the last observation carried forward (LOCF) method will be applied. PP (per protocol) analysis will be used for only the participants who have completed the entire course of the test exactly as specified in the trial plan. Primary evaluation of the intervention effect will be based on the results of the ITT analysis.

2.12. Participant Safety. During the informed consent procedures, study participants will be informed of potential adverse events such as local bleeding, subcutaneous hematoma, or pain associated with acupuncture treatment. Adverse events that occur after acupuncture treatment will be assessed at each visit and after treatment is concluded and will be recorded in the case report form (CRF). Appropriate actions will be taken to minimize adverse events. If a serious adverse event occurs, the blinding code will be broken and the case immediately reported to the clinical trial director and the Institutional Review Board (IRB) at Kyung Hee University Korean Medicine Hospital. If necessary, part or all of the trial will be suspended until further instructions are available. Should a participant experience adverse events from trial participation, she/he will be treated at the research institute's expense until recovery.

2.13. Confidentiality. After enrollment, all records that identify the research participants will be kept confidential and can only be viewed by researchers. All documents related to the study will be identified by unique codes rather than participants' names to protect confidentiality. All data and records related to the trial will be securely locked under the curation of the administration for three years after publication of the trial results and then destroyed.

2.14. Quality Control. All study researchers will receive centralized and unified training before the study. This study will be conducted under the periodic monitoring of AMSRC at Kyung Hee University and Korean Medicine Clinical Trial Center at Kyung Hee University Korean Medicine Hospital.
This protocol follows Standard Protocol Items: Recommendations for Interventional Trials (SPIRIT) (see Additional File 1 in Supplementary Materials) [47].

If a protocol modification is required due to a serious adverse event or unanticipated problem, the representatives of Kyung Hee University Korean Medicine Hospital and the AMSRC will discuss the matter fully to take the appropriate steps.

\section{Discussion}

The incidence of $\mathrm{AD}$ is increasing in developed countries, and the disorder affects both children and adults [2,3]. Conventional therapies, such as the use of topical corticosteroids, may be ineffective or lead to adverse side effects. Acupuncture treatment has been performed in $\mathrm{AD}$ patients, but controlled clinical trials to assess its effectiveness are lacking. In our pilot study [19], acupuncture was shown to be more effective in relieving the symptoms of $\mathrm{AD}$ than sham acupuncture, and the severity of $\mathrm{AD}$ was associated with the presence of GI symptoms.

The relationships between skin, brain, and GI system health [25] and between AD and the CNS [23, 24] suggest a role for acupuncture in relieving the symptoms of interrelated disorders of these organ systems. To assess this possible role, we designed a new confirmatory study with a complementary medicine protocol that builds on the results of our pilot study [19] and these studies.

We will recruit 36 mild-to-moderate AD patients for this study, scoring 30 to 80 points on a $100 \mathrm{~mm}$ VAS for pruritus ( 0 , no symptom at all; 10 , worst symptom ever) [27] and 10 to 40 points in the object SCORAD scores [28]. KM treatment such as acupuncture and herbal medicine may also relieve the symptoms of severe $\mathrm{AD}$ patients $[12,13]$. However, in this trial, patients will receive only acupuncture treatment. To remove factors that may confound or mask the effect of acupuncture, no antipruritic treatments such as topical steroids or antihistamines will be allowed. For participants' safety and convenience, patients with severe AD symptoms will be excluded. The difficulty of assessing relief from AD in patients with slight symptoms, who score less than 30 points on a $100 \mathrm{~mm}$ VAS for pruritus or less than 10 points in the object SCORAD scores, leads us to exclude them too.

In this study, we will use the same acupoints as in the pilot study [19]. These acupoints were selected based on traditional meridian theory [48], clinical experience, and consensus among experts in acupuncture and AD. Case studies using acupoints similar to this study (LI11, ST36, etc.) $[13,49]$ have shown relief of AD symptoms. The pilot study [19] showed significant improvement in itching and SCORAD score, further supporting our decision to use the same acupoints in the trial proposed here.

The dose of acupuncture treatment was also determined based on our pilot study [19], which compared two different doses of acupuncture treatment given two and three times weekly for four weeks, with the same doses of SA treatment. While both acupuncture groups significantly alleviated AD symptoms relative to the SA group, we found no significant 
difference between the twice- and thrice-weekly groups. To reduce the time and financial costs associated with hospital visits, we therefore decided that twice-weekly acupuncture treatment would be sufficient to detect treatment effects between VA and SA groups.

An appropriate sham control procedure is key to the success of an acupuncture RCT study. We designed the sham control to avoid physiological effects that might be induced by percutaneous needle insertion and to avoid specific acupoints. First, to avoid needle penetration, we will use the Park sham acupuncture device (AcuPrime Co., Ltd., Exeter, UK), which is known to be an adequate sham control for acupuncture [50], for administering both sham and real needles. Second, to avoid specific acupoints, we will use different acupoints in the two groups. Third, a blinding curtain will be used to ensure that acupuncture procedures take place out of sight of the participants. Last, a blinding questionnaire will also be used at the end of all acupuncture treatment sessions to assess effectiveness of blinding.

There is a battery of outcome measures in this study. They can be divided into five categories: (1) measures of the severity of AD symptoms: SCORAD, VAS (Pruritus), VAS (Insomnia), EASI, and POEM, (2) a measure of the QoL affected by AD: DLQI, (3) measures of the severity of dyspepsia symptoms: NDI-K, AR, VAS of dyspepsia, and abdominal PPT, (4) measures of central nervous system responses of $\mathrm{AD}$ patients: EEG, and (5) a credibility test, blinding test, and assessment of adverse effects.

In our previous pilot study [19], SCORAD (Total), VAS (Pruritus), VAS (Insomnia), POEM, DLQI, and credibility test scores differed significantly between the VA and SA groups over time. The changes in SCORAD (Objective), SCORAD (Total), and EASI were significantly different between the VA and SA groups and between weeks 0 and 4. SCORAD (Total) was the only indicator that differed both between groups and over time. Also, SCORAD is one of the most sensitive indicators for assessing the severity of $\mathrm{AD}$. Therefore, in this study, changes in SCORAD (Total) between weeks 0 and 4 are set as the primary outcome measure. SCORAD (Objective), VAS (Pruritus), VAS (Insomnia), EASI, DLQI, and POEM, which were significantly different between VA and SA groups in the pilot study, are set as the secondary outcomes.

The unique finding of our pilot study [19] was a significant effect of epigastric tenderness and dyspepsia on the main indicators of $\mathrm{AD}$, especially SCORAD, VAS (Pruritus), VAS (Insomnia), POEM, and DLQI. This supports the proposed "gut-brain-skin axis" linking skin disease with CNS and digestive symptoms [25]. Also, several studies have shown that $\mathrm{AD}$ patients with GI symptoms have significant improvement in the $\mathrm{AD}$ symptoms when their GI symptoms are treated $[51,52]$. For all these reasons, we added NDI-K, AR, VAS of dyspepsia, and abdominal PPT, which are quantitative indicators of indigestion, to the secondary outcomes of the trial that we propose here.

This study protocol will provide an important and thorough assessment of the effectiveness of twice-weekly acupuncture treatment in improving the symptoms of mildto-moderate $\mathrm{AD}$ in adults. It also aims to explore changes in
QoL affected by $\mathrm{AD}$, changes in the degree of GI symptoms, and EEG as secondary outcomes.

$\begin{array}{ll}\text { Abbreviations } \\ \text { AD: } & \text { Atopic Dermatitis } \\ \text { AMSRC: } & \text { Acupuncture and Meridian Science } \\ & \text { Research Center } \\ \text { AR: } & \text { Adequate Relief of functional dyspepsia } \\ & \text { pain and discomfort } \\ \text { DLQI: } & \text { Dermatology Life Quality Index } \\ \text { EASI: } & \text { Eczema Area and Severity Index } \\ \text { EEG: } & \text { Electroencephalogram } \\ \text { IA: } & \text { Intradermal Acupuncture } \\ \text { IRB: } & \text { Institutional Review Board } \\ \text { ITT analysis: } & \text { Intention-to-treat analysis } \\ \text { KM: } & \text { Korean Medicine } \\ \text { KMD: } & \text { Korean Medicine Doctor } \\ \text { MA: } & \text { Manual Acupuncture } \\ \text { NDI-K: } & \text { Nepean Dyspepsia Index-Korean version } \\ \text { POEM: } & \text { Patient Oriented Eczema Measure } \\ \text { PP analysis: } & \text { Per protocol analysis } \\ \text { PPT: } & \text { Pain Pressure Threshold } \\ \text { PTN: } & \text { Press Tack Needle } \\ \text { SA group: } & \text { Sham Acupuncture group } \\ \text { SCORAD: } & \text { Scoring Atopic Dermatitis } \\ \text { STRICTA: } & \text { Standards for Reporting Interventions in } \\ & \text { Clinical Trials of Acupuncture } \\ \text { QoL: } & \text { Quality of Life } \\ \text { VA group: } & \text { Verum Acupuncture group } \\ \text { VAS: } & \text { Visual Analog Scale. } \\ & \end{array}$

\section{Data Availability}

Data and materials are available upon request from the corresponding authors.

\section{Ethical Approval}

This study protocol was approved by the ethics committee at Kyung Hee University Korean Medicine Hospital (KOMCIRB-171117-HR-047,2018.3.23, ver1.4). It is performed in accordance with the standards of the International Council for Harmonisation of Good Clinical Practice and the revised version of the Declaration of Helsinki.

\section{Consent}

Before participation in the study, a study KMD would thoroughly inform all potential study participants about the study aims, methods, and the anticipated benefits and potential risks of participation. Patients who meet the inclusion criteria and are willing to participate would be included in the trial only after giving written informed consent. Signed written consent documents would be filed in the trial master file. 


\section{Disclosure}

This trial was registered via Clinical Research information Service (CRiS) on 13 April 2018 (identifier: KCT0002796).

\section{Conflicts of Interest}

The authors declare that they have no conflicts of interest.

\section{Authors' Contributions}

Younbyoung Chae, Hi-Joon Park, Hyangsook Lee, and Kyuseok Kim conceived and designed the trial. Jung Gun Park wrote the first draft. Jung Gun Park, Yu-Kang Kim, Younbyoung Chae, Hi-Joon Park, Hyangsook Lee, and Kyuseok Kim were responsible for critical revision of the draft. All authors read and approved the final manuscript.

\section{Acknowledgments}

The authors thank all subjects participating in the study. This research was supported by the National Research Foundation of Korea funded by the Korean Ministry of Science, ICT and Future Planning (NRF-2015M3A9E052338).

\section{Supplementary Materials}

SPIRIT 2013 Checklist: recommended items to address in a clinical trial protocol and related documents*. (Supplementary Materials)

\section{References}

[1] S. Weidinger and N. Novak, "Atopic dermatitis," The Lancet, vol. 387, no. 10023, pp. 1109-1122, 2016.

[2] H. Williams, C. Robertson, A. Stewart et al., "Worldwide variations in the prevalence of symptoms of atopic eczema in the international study of asthma and allergies in childhood," The Journal of Allergy and Clinical Immunology, vol. 103, no. 1, Pt 1, pp. 125-138, 1999.

[3] S. Nutten, "Atopic dermatitis: global epidemiology and risk factors," Annals of Nutrition and Metabolism, vol. 66, Suppl 1, pp. 8-16, 2015.

[4] L. F. Eichenfield, W. L. Tom, T. G. Berger et al., "Guidelines of care for the management of atopic dermatitis: section 2 . management and treatment of atopic dermatitis with topical therapies," Journal of the American Academy of Dermatology, vol. 71, no. 1, pp. 116-132, 2014.

[5] S. Park, H. Noh, C. Hwang et al., "Classification of atopic dermatitis into digestive and respiratory disorders on the basis of a literature study," The Journal of Korean Medicine Ophthalmology and Otolaryngology and Dermatology, vol. 29, no. 3, pp. 106-123, 2016.

[6] R. Sidbury, W. L. Tom, J. N. Bergman et al., "Guidelines of care for the management of atopic dermatitis: section 3 . Management and treatment with phototherapy and systemic agents," Journal of the American Academy of Dermatology, vol. 71, no. 2, pp. 327-349, 2014.
[7] E. Roekevisch, P. I. Spuls, D. Kuester, J. Limpens, and J. Schmitt, "Efficacy and safety of systemic treatments for moderate-tosevere atopic dermatitis: a systematic review," The Journal of Allergy and Clinical Immunology, vol. 133, no. 2, pp. 429-438, 2014.

[8] J. Ring, A. Alomar, T. Bieber et al., "Guidelines for treatment of atopic eczema (atopic dermatitis) Part II," Journal of the European Academy of Dermatology and Venereology, vol. 26, no. 9, pp. 1176-1193, 2012.

[9] R. B. Vender, "Alternative treatments for atopic dermatitis: a selected review.," Skin Therapy Letter, vol. 7, no. 2, pp. 1-5, 2002.

[10] F. J. Bath-Hextall, C. Jenkinson, R. Humphreys, and H. C. Williams, "Dietary supplements for established atopic eczema," Cochrane Database of Systematic Reviews, vol. 2, Article ID CD005205, 2012.

[11] R. J. Boyle, F. J. Bath-Hextall, J. Leonardi-Bee, D. F. Murrell, and M. L.-K. Tang, "Probiotics for the treatment of eczema: a systematic review," Clinical \& Experimental Allergy, vol. 39, no. 8, pp. 1117-1127, 2009.

[12] Y. Yun, J. Son, K. Kim, B. Jang, I. Choi, and S. Ko, "Effectiveness and safety of combination treatment of herbal medicines and oral antihistamines for atopic dermatitis: a retrospective chart review," Integrative Medicine Research, vol. 6, no. 1, pp. 19-25, 2017.

[13] Y. Yun, S. Lee, S. Kim, and I. Choi, "Inpatient treatment for severe atopic dermatitis in a traditional korean medicine hospital: introduction and retrospective chart review," Complementary Therapies in Medicine, vol. 21, no. 3, pp. 200-206, 2013.

[14] K. C. Lee, A. Keyes, J. R. Hensley et al., "Effectiveness of acupressure on pruritus and lichenifi cation associated with atopic dermatitis: a pilot trial," Acupuncture in Medicine, vol. 30, no. 1, pp. 8-11, 2012.

[15] X. H. Quan, S. R. Cheng, H. Ma, H. X. Huang, B. Wang, and X. H. Chen, "Combination of flying needle with chinese herbal medicine in the treatment of atopic dermatitis: a clinical trial," Pakistan Journal of Pharmaceutical Sciences, vol. 27, no. 5, pp. 1687-1690, 2014.

[16] C. Yu, P. Zhang, Z. T. Lv et al., "Efficacy of acupuncture in itch: a systematic review and meta-analysis of clinical randomized controlled trials," Evidence-Based Complementary and Alternative Medicine, vol. 2015, Article ID 208690, 5 pages, 2015.

[17] J.-B. Han, C. W. Kim, B. Sun et al., "The antipruritic effect of acupuncture on serotonin-evoked itch in rats," Acupuncture \& Electro-Therapeutics Research, vol. 33, no. 3-4, pp. 145-156, 2008.

[18] H. Y. Tan, G. B. Lenon, A. L. Zhang, and C. C. Xue, "Efficacy of acupuncture in the management of atopic dermatitis: a systematic review," Clinical and Experimental Dermatology, vol. 40, no. 7, pp. 711-715, 2015.

[19] S. Kang, Y.-K. Kim, M. Yeom et al., "Acupuncture improves symptoms in patients with mild-to-moderate atopic dermatitis: A randomized, sham-controlled preliminary trial," Complementary Therapies in Medicine, vol. 41, pp. 90-98, 2018.

[20] K. Dal, O. S. Deveci, M. Kucukazman et al., "Decreased parasympathetic activity in patients with functional dyspepsia," European Journal of Gastroenterology \& Hepatology, vol. 26, no. 7, pp. 748-752, 2014.

[21] M. K. Boettger, K.-J. Bär, A. Dohrmann et al., "Increased vagal modulation in atopic dermatitis," Journal of Dermatological Science, vol. 53, no. 1, pp. 55-59, 2009.

[22] C. Uchida, H. Waki, Y. Minakawa, H. Tamai, T. Hisajima, and K. Imai, "Evaluation of autonomic nervous system function 
using heart rate variability analysis during transient heart rate reduction caused by acupuncture," Medical Acupuncture, vol. 30, no. 2, pp. 89-95, 2018.

[23] A. Alexopoulos and G. P. Chrousos, "Stress-related skin disorders," Reviews in Endocrine and Metabolic Disorders, vol. 17, no. 3, pp. 295-304, 2016.

[24] V. Napadow, A. Li, M. L. Loggia et al., "The brain circuitry mediating antipruritic effects of acupuncture," Cerebral Cortex, vol. 24, no. 4, pp. 873-882, 2014.

[25] P. Arck, B. Handjiski, E. Hagen et al., "Is there a 'gut-brain-skin axis'?” Experimental Dermatology, vol. 19, no. 5, pp. 401-405, 2010.

[26] L. F. Eichenfield, W. L. Tom, S. L. Chamlin et al., "Guidelines of care for the management of atopic dermatitis: section 1 . Diagnosis and assessment of atopic dermatitis," Journal of the American Academy of Dermatology, vol. 70, no. 2, pp. 338-351, 2014.

[27] S. Takeuchi, H. Saeki, S. Tokunaga et al., "A randomized, openlabel, multicenter trial of topical tacrolimus for the treatment of pruritis in patients with atopic dermatitis," Annals of Dermatology, vol. 24, no. 2, pp. 144-150, 2012.

[28] A. P. Oranje, "Practical issues on interpretation of scoring atopic dermatitis: SCORAD index, objective SCORAD, patientoriented SCORAD and three-item severity score," Pathogenesis and Management of Atopic Dermatitis, vol. 41, pp. 149-155, 2011.

[29] J. Jang, J. Kim, S. Lee, and K. H. Kim, "A study on the development of the abdomen diagnosis devices based on traditional Korean medicine," The Journal of Korean Institute of Communications and Information Sciences, vol. 42, no. 7, pp. 1493-1501, 2017.

[30] G. Ricci, A. Dondi, and A. Patrizi, "Useful tools for the management of atopic dermatitis," American Journal of Clinical Dermatology, vol. 10, no. 5, pp. 287-300, 2009.

[31] J. F. Stalder, A. Taieb, D. J. Atherton et al., "Severity scoring of atopic dermatitis: the SCORAD index. consensus report of the european task force on atopic dermatitis," Dermatology, vol. 186, no. 1, pp. 23-31, 1993.

[32] J. Schmitt, S. Langan, S. Deckert et al., "Assessment of clinical signs of atopic dermatitis: A systematic review and recommendation," The Journal of Allergy and Clinical Immunology, vol. 132, no. 6, pp. 1337-1347, 2013.

[33] R. Chopra, P. P. Vakharia, R. Sacotte et al., "Relationship between EASI and SCORAD severity assessments for atopic dermatitis," British Journal of Dermatology, vol. 172, no. 5, pp. 1353-1357, 2015.

[34] C. R. Charman, A. J. Venn, and H. C. Williams, "The patientoriented eczema measure: Development and initial validation of a new tool for measuring atopic eczema severity from the patients' perspective," JAMA Dermatology, vol. 140, no. 12, pp. 1513-1519, 2004.

[35] A. Y. Finlay and G. K. Khan, "Dermatology life quality index (DLQI) - a simple practical measure for routine clinical use," Clinical and Experimental Dermatology, vol. 19, no. 3, pp. 210216, 1994.

[36] H. Both, M.-L. Essink-Bot, J. Busschbach, and T. Nijsten, "Critical review of generic and dermatology-specific healthrelated quality of life instruments," Journal of Investigative Dermatology, vol. 127, no. 12, pp. 2726-2739, 2007.

[37] K. H. Cho, K. Toshiaki, T. Katsutoshi et al., "An introduction of abdominal palpation signs in japanese oriental medicine -a new approach to the practice of abdominal palpation in korean oriental medicine," Journal of Internal Korean Medicine, vol. 18, no. 1, pp. 86-95, 1997.

[38] S. J. Ko, H. J. Lee, S. K. Kim et al., "Development of the quantitative indicator of abdominal examination for clinical application: a pilot study," The Journal of Alternative and Complementary Medicine, vol. 21, no. 6, pp. 358-363, 2015.

[39] N. J. Talley, M. Haque, J. W. Wyeth et al., "Development of a new dyspepsia impact scale: the Nepean Dyspepsia Index," Alimentary Pharmacology \& Therapeutics, vol. 13, no. 2, pp. 225235, 1999.

[40] N. J. Talley, M. Verlinden, and M. Jones, "Quality of life in functional dyspepsia: responsiveness of the nepean dyspepsia index and development of a new 10-item short form," Alimentary Pharmacology \& Therapeutics, vol. 15, no. 2, pp. 207-216, 2001.

[41] S. Y. Lee, S. C. Choi, Y. K. Cho, and M. G. Choi, "Validation of the Nepean dyspepsia index- Korean version," Journal of Neurogastroenterology and Motility, vol. 9, suppl 48S, 2003.

[42] N. J. Talley, S. V. Van Zanten, L. R. Saez et al., "A doseranging, placebo-controlled, randomized trial of alosetron in patients with functional dyspepsia," Alimentary Pharmacology \& Therapeutics, vol. 15, no. 4, pp. 525-537, 2001.

[43] C. Vincent, "Credibility assessment in trials of acupuncture," Complementary Medicine Research, vol. 4, pp. 8-11, 1990.

[44] Z. Qin, Y. Ding, J. Wu et al., "Efficacy of acupuncture for degenerative lumbar spinal stenosis: protocol for a randomised sham acupuncture-controlled trial," BMJ Open, vol. 6, no. 11, Article ID e012821, 2016.

[45] H. Holle, K. Warne, A. K. Seth, H. D. Critchley, and J. Ward, "Neural basis of contagious itch and why some people are more prone to it," Proceedings of the National Acadamy of Sciences of the United States of America, vol. 109, no. 48, pp. 19816-19821, 2012.

[46] J. A. Pineda, "The functional significance of mu rhythms: translating 'seeing' and 'hearing' into 'doing'” Brain Research Reviews, vol. 50, no. 1, pp. 57-68, 2005.

[47] A.-W. Chan, J. M. Tetzlaff, P. C. Gøtzsche et al., "SPIRIT 2013 explanation and elaboration: guidance for protocols of clinical trials," BMJ, vol. 346, Article ID e7586, 2013.

[48] Korean Acupuncture \& Moxibustion Medicine Society, Acupuncture Medicine, Hanmi Medical, Seoul, Korea, 2014.

[49] M. Kim, Y. Yun, K. S. Kim, and I. Choi, “Three cases of atopic dermatitis in pregnant women successfully treated with Korean medicine," Complementary Therapies in Medicine, vol. 21, no. 5, pp. 512-516, 2013.

[50] C.-W. Tan, P. Sheehan, and D. Santos, "Discrimination accuracy between real and sham needles using the Park sham device in the upper and lower limbs," Acupuncture in Medicine, vol. 29, no. 3, pp. 208-214, 2011.

[51] T. Park, "Effect of the herbal medicine treatment for gastrointestinal dysfunction in children with atopic dermatitis," Complementary Alternative Medicine Graduate School of Complementary Alternative Medicine Pocheon CHA University, pp. $1-48,2008$.

[52] H. Noh, S. Park, I. Park, E. Jo, and M. Park, “Two cases of atopic dermatitis with gastrointestinal disorders treated with soshiho-tang," The Journal of Korean Medicine Ophthalmology and Otolaryngology and Dermatology, vol. 29, no. 3, pp. 208216, 2016. 


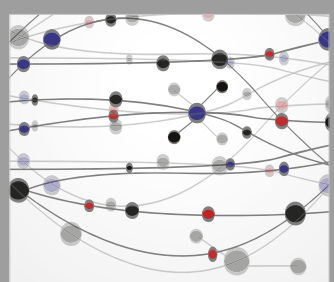

The Scientific World Journal
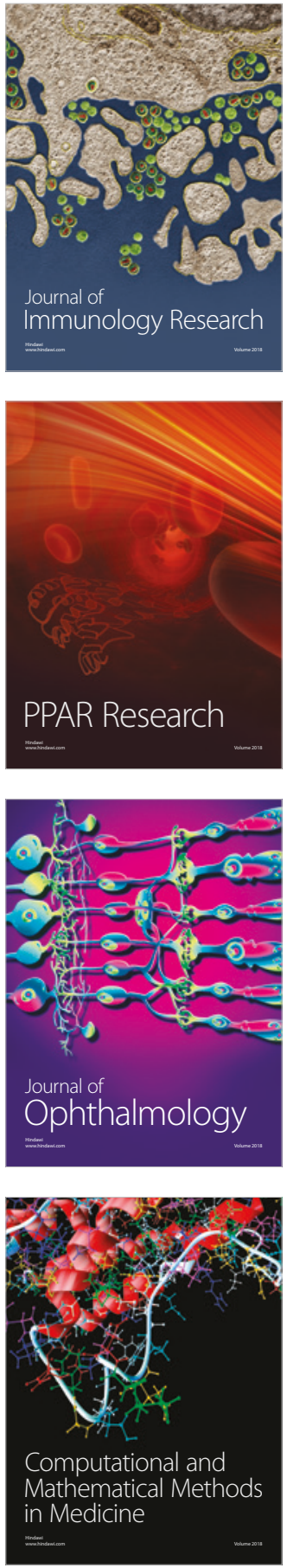

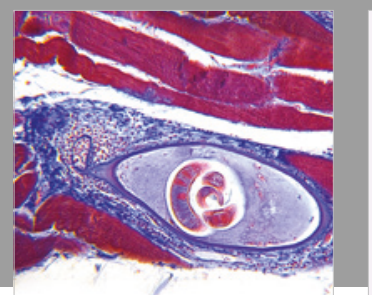

Gastroenterology Research and Practice

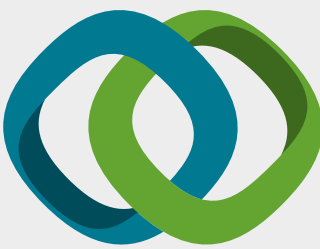

\section{Hindawi}

Submit your manuscripts at

www.hindawi.com
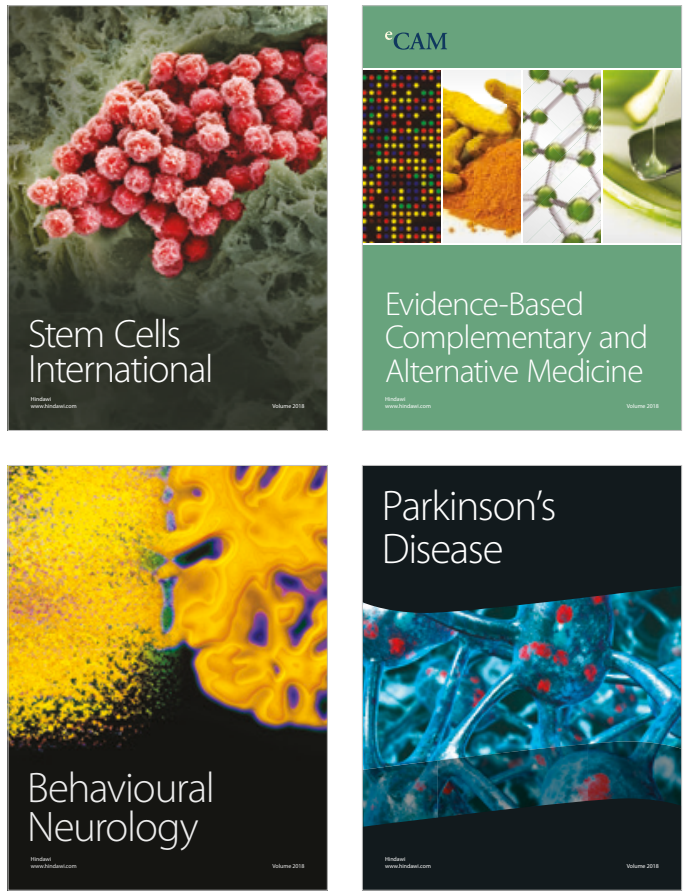

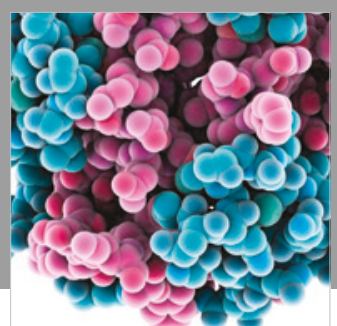

ournal of

Diabetes Research

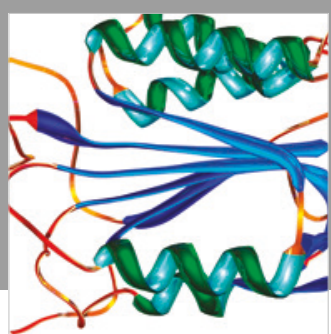

Disease Markers
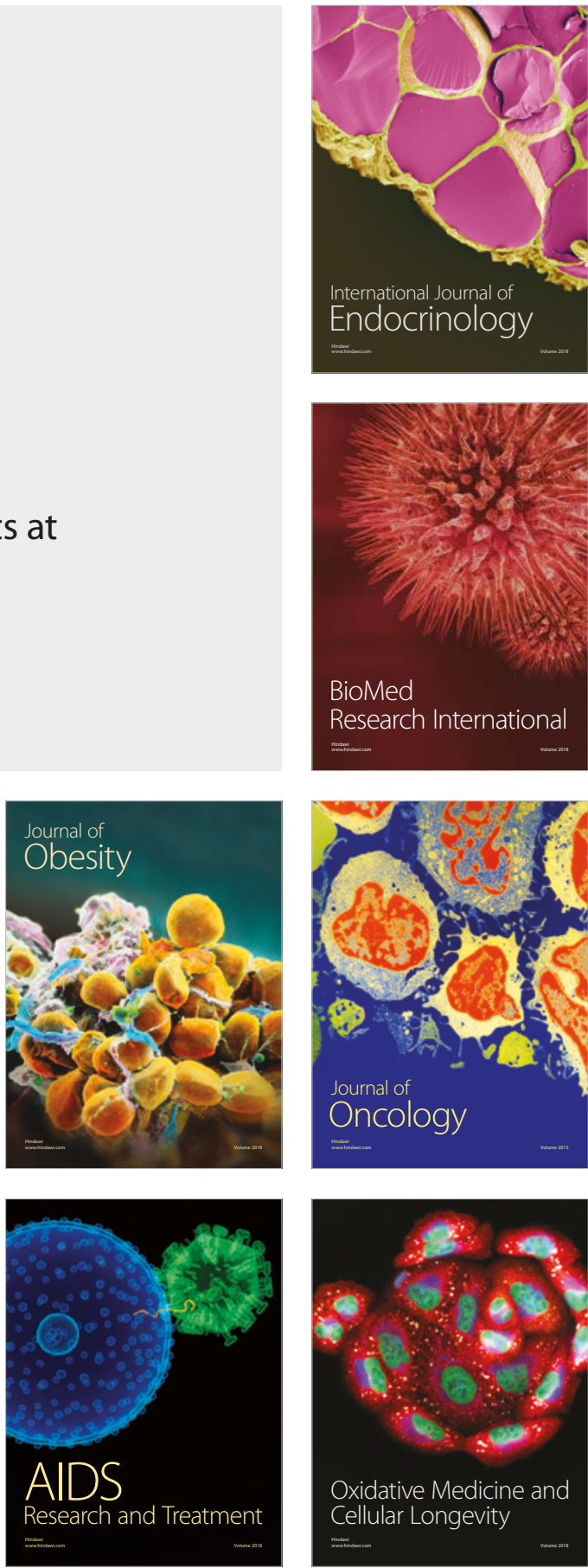